\title{
PANORAMA DA COMUNICAÇÃO CIENTÍFICA DOS DOCENTES-PESQUISADORES DO CAMPO DA CANCEROLOGIA BRASILEIRA ${ }^{1}$
}

\author{
Kátia de Oliveira Rodrigues \\ Doutora em Ciência da Informação pela Universidade \\ Federal de Minas Gerais (UFMG). Professora do \\ Programa de Pós-Graduação do Instituto de Ciência da \\ Informação da Universidade Federal da Bahia (UFBA). \\ katiarodrigues10@gmail.com \\ https://orcid.org/0000-0002-4909-8745
}

\author{
Marlene Oliveira \\ Doutora pela Universidade de Brasília (UnB). Professora \\ da Escola de Ciência da Informação - ECI da \\ Universidade Federal de Minas Gerais (UFMG) \\ marleneotmelo@gmail.com \\ https://orcid.org/0000-0003-2834-1272
}

\section{RESUMO}

A comunicação científica consiste em um processo cíclico, que envolve diferentes etapas, a exemplo da produção e da publicação dos resultados da pesquisa. O presente artigo tem como objetivo caracterizar a produção científica dos docentes-pesquisadores dos programas de pós-graduação, no campo da Cancerologia brasileira, no período 2005-2015. A pesquisa caracteriza-se como descritiva, com abordagem quantitativa. Os resultados evidenciaram padrões de produção científica, a exemplo de: predominância de artigo de periódico científico, publicação em inglês e artigos publicados em periódicos com Qualis/Capes A1, A2 e B1, na avaliação de 2013-2016.

Palavras-chave: Comunicação científica. Produção científica. Canal de comunicação.

\section{PANORAMA OF THE SCIENTIFIC COMMUNICATIONTEACHERS-RESEARCHERS IN THE FIELD OF BRAZILIAN CANCEROLOGY}

\begin{abstract}
Scientific communication consists of a cyclical process, which involves different stages, such as production and publication of research results. The present paper aims at characterizing the scientific production of professors-researchers in graduate programs, in the field of Brazilian Cancerology, in the period 2005-2015. The research is characterized as descriptive, with a quantitative approach. Results showed patterns of scientific production, such as: predominance of scientific journal papers, publication in English and publication in journals with Qualis/Capes $A 1, A 2$, and $B 1$, taking the 2013-2016 evaluation as a reference.
\end{abstract}

Keywords Scientific communication. Scientific production. Communication channel.

Recebido em: 21/04/2020

Aceito em: 22/08/2020

Publicado em: 11/09/2020

\section{INTRODUÇÃO}

A comunicação, como esclarece Garvey (1979, p. ix, tradução nossa), em seu livro Communication: the essence of science, é "[...] o espectro completo de atividades associadas com a produção, difusão e uso de informações desde que o cientista tem uma ideia para sua pesquisa até as informações sobre os resultados desta pesquisa

\footnotetext{
${ }^{1} \mathrm{O}$ referido artigo é um recorte da tese intitulada Fatores que influenciam o comportamento de citação de docentes-pesquisadores do campo da Cancerologia brasileira, defendida no Programa de Pós-Graduação em Ciência da Informação na Universidade Federal de Minas Gerais..
} 
serem aceitos como um componente do conhecimento científico." Como o próprio título do livro indica, o autor considera a comunicação como "a essência da ciência", como o meio através do qual a ciência materializa-se, desde a concepção de um problema de pesquisa até o processo de produção e publicação dos resultados. A inexistência da comunicação não apenas fragiliza a ciência como também a torna estagnada, no tempo e no espaço.

Neste contexto, o problema de pesquisa deste artigo pode traduzir-se na seguinte questão: como se vem caracterizando a produção científica dos docentes-pesquisadores dos programas de pós-graduação, no campo da Cancerologia brasileira, no período 2005 a 2015? Isto posto, a pesquisa tem como objetivo geral compreender como vem se caracterizando a produção científica dos docentes-pesquisadores dos programas de pós-graduação, no campo da Cancerologia brasileira, no período de 2005 a 2015. Para alcançá-lo, foram definidos como objetivos específicos: a) caracterizar a produção científica dos docentes-pesquisadores do campo investigado e b) identificar os títulos de periódicos mais utilizados, como canal de comunicação, pelos docentes-pesquisadores e o respectivo estrato no Qualis/Capes (2013-2016).

\section{REFLEXÕES ACERCA DA PUBLICAÇÃO DA CIÊNCIA}

Comunicar os resultados de uma pesquisa é tornar conhecido e reconhecido um esforço de produção que não tem mérito de ciência, exceto na relação com o campo. Quando vêm a público os resultados de uma determinada pesquisa, estes dialogam com o conhecimento já acumulado pelos pesquisadores do campo e servem tanto de ponto de partida para novas pesquisas quanto como interlocutores em relação a outros resultados de pesquisa, retroalimentando o processo cíclico da comunicação científica.

Entretanto, vale ressaltar que, nem tudo que é produzido cientificamente é aceito pelos pares, ficando, assim, impossibilitado de alcançar o objetivo da ciência, que, segundo Ziman (1979, p. 24), consiste em "[...] alcançar um consenso de opinião racional que abranja o mais vasto campo possível". Assim, a informação científica precisa do reconhecimento e da aprovação dos pares, no campo científico. Bourdieu (2014, p. 101) também aponta para a necessidade de reconhecimento pelos pares.

O conhecimento científico é aquilo que sobreviveu às objeções e pode resistir às objeções futuras. A opinião validada é aquela que é reconhecida, pelo menos negativamente, porque já não suscita objeções 
pertinentes ou não tem melhor explicação. Nestas lutas que aceitam como árbitro o veredicto da experiência, ou seja, daquilo que os investigadores concordam em considerar como real, a verdade é o conjunto das representações consideradas verdadeiras por serem produzidas segundo as regras que definem a produção da verdade; é nisto que concordam os concorrentes que estão de acordo sobre os princípios de verificação, sobre os métodos comuns de validação das hipóteses.

Ainda no contexto do movimento circular da comunicação científica, Garvey (1979) elucida que a informação, na ciência, evolui paulatinamente com a incorporação de novas informações. Este processo de reabastecimento de informação, na comunidade ${ }^{2}$ científica, demanda que seja disseminada por um canal formal, o qual "[...] encontra-se disponível por longos períodos de tempo para um público amplo" (MEADOWS,1999, p. 7), possibilitando que outros pesquisadores possam utilizar aquela pesquisa. Rompendo as barreiras do tempo e do espaço, o canal formal opõe-se ao informal, que circula no espaço restrito do público especializado.

Os canais informais e formais não são utilizados de forma análoga por toda a comunidade científica.(LETA, 2005; MUELLER, 2005, 2007a, 2007b, 2008). Pesquisas a exemplo das realizadas por Leta (2005), Mueller (2005, 2007a, 2007b, 2008) e Nederhof (2006) evidenciaram que o artigo científico é empregado nas Ciências Naturais, Exatas e Biológicas.Os anais de eventos científicos, por sua vez,são um canal de disseminação dos resultados de pesquisas nas Engenharias e Tecnologias, e o livro é o principal canal de comunicação das Ciências Sociais e Humanas, assim como o artigo científico.

Acerca da especificidade do canal de publicação escolhido pelas diferentes áreas de conhecimento, é Mueller (2005) quem explica que, no caso específico das Ciências Normais ou Experimentais, por exemplo, as pesquisas são realizadas em equipes e os resultados são disseminados em artigos concisos, publicados em "[...] periódicos de circulação internacional e em língua inglesa". (MUELLER, 2005).

Apesar da variedade de canais de comunicação disponíveis para disseminação dos resultados das pesquisas, observa-se que o periódico científico vem predominando em um número significativo de campos investigativos, como o mais relevante. Em decorrência dessa representatividade, provavelmente a qualidade do periódico científico tenha se constituído como um dos indicadores de avaliação dos Programas de Pós-Graduação brasileira, desde 1998, pela Coordenação de Aperfeiçoamento de Pessoal de Nível Superior (Capes).

\footnotetext{
${ }^{2}$ Neste artigo manteve-se o termo "comunidade" nos casos em que o autor citado utiliza-se deste conceito e não do conceito de campo.
} 
Inicialmente, a avaliação pela Capes consistia em classificar, conforme os critérios definidos pelos membros da comissão avaliadora de cada área do conhecimento, os títulos de periódicos científicos, a partir de seis categorias: em relação ao nível ( $A$ - alta, $B$ - média, $C$ - baixa) e no que se refere à circulação ( 1 - internacional, 2 - nacional e 3 - local). A combinação destas seis categorias resultava em nove categorias distintas, A1; A2; A3; B1; B2; B3; C1; C2 e C3. (BARATA, 2016; MUGNAINI, 2011).

Posteriormente, na avaliação Capes, do período de 2007 a 2009, uma nova sistemática de classificação passou a vigorar, para a avaliação dos títulos de periódicos científicos. A escala de classificação divide-se em sete estratos: A1; A2; B1; B2; B3; B4; B5 e C. (CAMPOS, 2010; COORDENAÇÃO DE APERFEIÇOAMENTO DE PESSOAL DE NÍVEL SUPERIOR, 2004; ROCHA-E-SILVA, 2009). Assim, o estrato A1 equivaleria à classificação que fosse maior, enquanto que o C corresponderia ao estrato de menor qualidade. Como o Qualis/Capes é o reflexo de cada área e os critérios de avaliação são definidos pelos seus membros, pode ocorrer de um mesmo título de periódico ser classificado em diferentes estratos, conforme a área de avaliação.

Quando implantado, o Qualis/Capes tinha como finalidade a classificação dos periódicos científicos utilizados para tornar pública a produção científica dos docentes-pesquisadores e discentes dos programas de pós-graduação brasileira. Posteriormente, foram incorporadas mais duas finalidades ao Qualis/Capes: estimular a disseminação do programa de pós-graduação em periódicos científicos representativos para cada área e a indicação, pela comissão de área, de periódicos relevantes, mesmo que até o período da avaliação não tenham sido informados, na Coleta Capes. (COORDENAÇÃO DE APERFEIÇOAMENTO DE PESSOAL DE NÍVEL SUPERIOR, 2004).

A aplicação do Qualis/Capes, embora consolidada, permanece envolta em controvérsias, a exemplo dos impactos nos periódicos científicos nacionais na área de Medicina II e III, após as mudanças do Qualis/Capes para o período de 2007 a 2009. De acordo com o editorial do Jornal Brasileiro de Pneumologia, o qual foi assinado por 60 (sessenta) editores dos principais periódicos científicos médicos do país, entre as críticas elencadas pelos autores está o "[...] receio de que os novos critérios da Capes possam criar uma subclasse de periódicos baseada exclusivamente no fator de impacto ISI.". Os autores complementam que "[...] os novos critérios além de considerarem apenas o fator de impacto, estabeleceram pontos de corte bem mais elevados.". (ANDRIOLO et al., 2010, p. 1). 


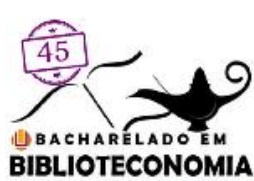

Campos (2010) faz uma análise crítica, em relação aos critérios de classificação do Qualis/Capes, na área das Engenharias I. Para o autor, "[...] o fator de impacto, como indicador cienciométrico,foi fortemente utilizado na classificação dos periódicos dos estratos mais elevados". (CAMPOS, 2010, p. 477). Como resultado, obteve-se duas perspectivas: na primeira, a classificação em estratos de baixo impacto dos títulos de periódicos, predominantemente nacionais, pode instigar seus editores a melhorar a qualidade de seus periódicos e, assim, avançar nos estratos; na segunda, que esses critérios de avaliação dos títulos de periódicos, na referida área, podem afastar os pesquisadores reconhecidos daqueles periódicos nacionais, classificados em estrato de baixo impacto, em favor dos nacionais de alto impacto e dos internacionais. (CAMPOS, 2010).

As análises realizadas por estes autores estão diretamente relacionadas ao fator de impacto, e precisam ser observadas construtivamente, tendo em vista que entre os objetivos a serem alcançados pelos programas de pós-graduação brasileira está a internacionalização. Conquistar este status depende de vários fatores, entre os quais, que as produções dos docentes-pesquisadores e discentes dos programas sejam reconhecidos pelos pares-concorrentes internacionais, através das citações (COORDENAÇÃO DE APERFEIÇOAMENTO DE PESSOAL DE NÍVEL SUPERIOR, 2016). Para tanto, dois são os caminhos que podem ser percorridos pelos docentes-pesquisadores brasileiros: primeiro, ter os artigos aceitos em periódicos científicos de alto fator de impacto e, segundo, melhorar a qualidade dos periódicos científicos nacionais, tornando-os competitivos entre os pesquisadores nacionais e internacionais, aumentando assim sua visibilidade e possibilidade de serem lidos e citados.

\section{PROCEDIMENTOS METODOLÓGICOS}

A pesquisa caracteriza-se como descritiva quanto aos objetivos, com abordagem quantitativa. O corpus da pesquisa foi composto pelas produções dos docentes, pesquisadores de programas de pós-graduação, no campo da Cancerologia brasileira: PPG-FAP; PPG-HCB; PPG-INCA; PPG-UFPA; PPG-USP e PPG-USP/RP. Quanto ao recorte temporal, decidiu-se investigar o período de 2005 a 2015.

A coleta dos dados aconteceu em quatro etapas: a primeira etapa efetivou-se com uma busca através do link "Docentes", da Plataforma Sucupira e dos Cadernos de Indicadores da Capes, para identificar e listar os docentes-pesquisadores dos programas 
de pós-graduação, no campo da Cancerologia brasileira, em uma planilha eletrônica Microsoft Excel. A segunda etapa efetivou-se com a consulta no link "Buscar currículo", da Plataforma Lattes, do CNPq, quando foram recuperados os Currículos Lattes dos docentes-pesquisadores dos programas em análise. Após a localização do currículo, este era salvo em uma pasta denominada "Currículos dos docentes-pesquisadores", no formato de arquivo Portable Document Format (PDF). Nesta etapa, foram mapeadas as informações da produção científica desses profissionais, no período estabelecido. Estas informações foram lançadas na planilha utilizada na primeira etapa, complementando, assim, os dados referentes aos docentes-pesquisadores. Antes de iniciar a etapa seguinte, da coleta dos dados, decidiu-se retirar da planilha as produções repetidas, resultantes de pesquisas realizadas em coautoria com docentes-pesquisadores dos programas em análise.

$\mathrm{Na}$ etapa seguinte, a terceira, realizou-se pesquisa na Web of Science (WoS) para identificar e coletar informações sobre as produções científicas dos docentes-pesquisadores, informadas no Currículo Lattes. Justifica-se a escolha pela potencialidade desta base de dados para a ciência e por possibilitar a migração dos dados para o HistCite TM, versão 12.03.1756. Nesta etapa, recorreu-se à "Principal Coleção da Web of Science", de modo a acionarmos estratégias de busca no link "Pesquisa Básica". Informou-se no campo "Tempo Estipulado" o período de 2005-2015, para refinar a busca na base. Em seguida, as informações referentes à produção científica dos docentes-pesquisadores, recuperadas na WoS, eram armazenadas na pasta Downloads. Estas informações, em seguida, eram salvas em formato bloco de notas, na pasta denominada FAKEPATH. A quarta etapa da coleta de dados baseou-se na migração dos metadados armazenados na pasta FAKEPATH, para o HistCite TM, versão 12.03.17, gerando um banco de dados denominado "HistCite - Produção dos docentes-pesquisadores (2005-2015)".

Quanto à análise dos dados, optou-se por descrever as seguintes categorias: tipologias das publicações dos docentes-pesquisadores; tipo de autoria; idioma das produções; ano de publicação; produções dos docentes-pesquisadores por programas de pós-graduação; publicações por docentes-pesquisadores; docentes-pesquisadores com maior número de publicações; estrato do Qualis/Capes dos títulos de periódicos utilizados como canal de comunicação pelos docentes-pesquisadores e títulos de periódicos mais utilizados como canal de comunicação, pelos docentes-pesquisadores. 


\section{PUBLICAÇÕES DOS DOCENTES-PESQUISADORES}

Primeiramente, listaram-se 11.639 produções. Contudo, destas, 3.444 (29,6\%) foram descartadas, porque eram duplicatas, originárias de pesquisas realizadas em coautoria entre docentes-pesquisadores do campo da Cancerologia brasileira e $2.138(18,4 \%)$ não se encontravam indexadas na WoS. Assim, o total das produções científicas dos docentes-pesquisadores analisados correspondeu a 6.057 (52,0\%), do geral levantado.

Um dos aspectos importantes a analisar nas pesquisas sobre comportamento de citação é a tipologia das publicações no campo científico, por duas razões. Primeiro, porque cada campo científico apresenta particularidades quanto ao canal de comunicação científica a ser utilizado pelos pesquisadores, para tornar públicos os resultados de suas pesquisas. Pesquisadores como Mueller (2005, 2007a, 2007b, 2008), Mugnaini, Meneghini e Packer (2007) e Nederhof (2006), por exemplo, constataram que a utilização dos diferentes tipos de canais de comunicação, como livro, anais de evento, artigo de periódico, entre outros, se diferencia,conforme o campo científico. Estas variações podem estar associadas a diferentes fatores, dentre os quais, o fluxo de informação demandado pelos pesquisadores do campo. Esse fator mostra-se bastante relevante no campo da Cancerologia, já que a agilidade do fluxo informacional é uma exigência.

Segundo, porque a tendência é que a tipologia das publicações empregadas pelos pesquisadores do campo científico para disseminar os resultados das pesquisas retroalimente o processo de comunicação científica. Assim, em função destas particularidades, em geral, os resultados referentes às tipologias identificadas nas publicações de pesquisadores de um campo são reproduzidos nas citações daqueles. O Gráfico 1 possibilita uma análise geral das tipologias presentes nas publicações dos docentes-pesquisadores. 
Gráfico 1 - Tipologia das publicações dos docentes-pesquisadores (2005-2015)

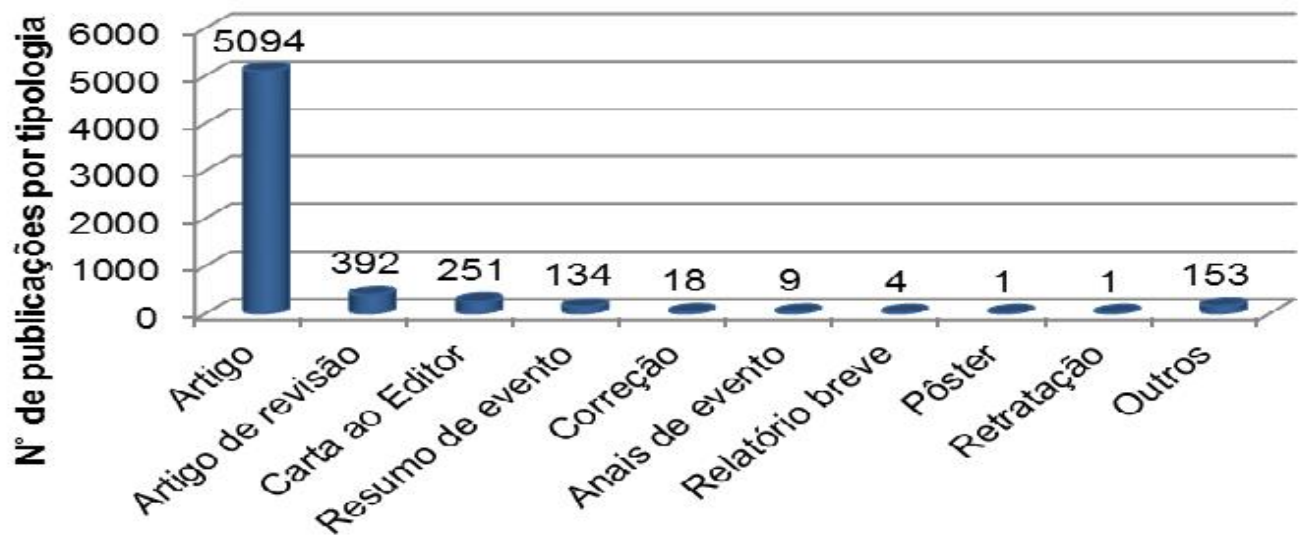

Tipologia das publicaçöes dos docentes-pesquisadores

Fonte: Dados da pesquisa.

No campo da Cancerologia brasileira, o "Artigo"3 apresentou uma ocorrência expressiva em comparação com as demais tipologias das publicações dos docentes-pesquisadores deste campo. Este resultado confirma a tendência da ciência contemporânea de que a maioria dos campos prefere o artigo de periódico para disseminar seus resultados de pesquisa. Isso explica-se por ser um canal rápido, que não demanda tanto tempo quanto o livro, por exemplo. Do total de 6.057 publicações dos docentes-pesquisadores, 5.094 (84,1\%) eram "artigo", ao passo que as tipologias "anais de evento"; "relatório breve"; "pôster" e "retratação" obtiveram as menores frequências, 9 (0,14\%); 4 (0,07\%); 1 (0,02\%); 1 (0,02\%). No item "Outros", encontram-se 152 (2,5\%) "editoriais" e 1 (0,02\%) "notícias".

Em relação ao tipo de autoria presente nas produções dos docentes-pesquisadores, no campo da Cancerologia brasileira, os resultados da pesquisa revelam a predominância da autoria múltipla, com $5.991(99,0 \%)$ produções, enquanto que as produções com autoria individual totalizam 66 (1,0\%). Como ilustra o Gráfico 2.

\footnotetext{
${ }^{3}$ Para análise das tipologias das produções dos docentes-pesquisadores, a categoria "Artigo" refere-se a "parte de uma publicação com autoria declarada, que apresenta e discute ideias, métodos, técnicas, processos e resultados nas diversas áreas do conhecimento.", podendo ser de temáticas ou abordagens originais. Enquanto que a categoria "Artigo de revisão" destina-se a "parte de uma publicação que resume, analisa e discute informações já publicadas.” (ASSOCIAÇÃO BRASILEIRA DE NORMAS TÉCNICAS, 2003, p. 2).
} 
Gráfico 2 - Tipo de autoria das produções dos docentes-pesquisadores (2005-2015)

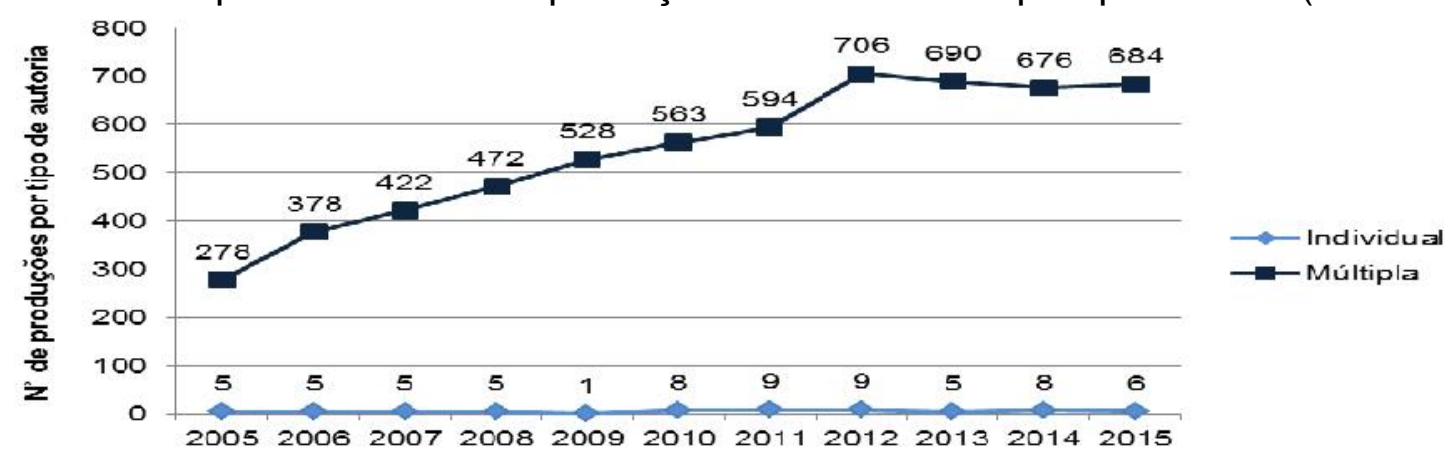

Fonte: Dados da pesquisa.

Apesar do crescimento importante de produções com autoria múltipla, no período de 2005 a 2012, registra-se um tênue declínio, a partir de 2013. Quanto à frequência de produções com autoria individual, observa-se uma oscilação, durante todo o período em análise, 2005-2015, com uma taxa que não ultrapassa o número de 9 produções. Sobre autoria múltipla, Bufrem (2009) esclarece que o trabalho em equipe

"[...] pode representar uma tendência já verificada na literatura mundial, a de estar a ciência cada vez mais sendo desenvolvida em equipe [...] principalmente pelas possibilidades de intercâmbios interdepartamentais e interinstitucionais [...]".

Essa tendência está explicitada nas orientações da comissão de avaliação de Medicina I, da Coordenação de Aperfeiçoamento de Pessoal de Nível Superior (2016), ao sinalizar a necessidade da presença de pesquisadores de diversas subáreas, contribuindo para a realização de pesquisa. Além disso, a própria caraterística do objeto de pesquisa no campo em estudo exige do docente-pesquisador colaboração científica nacional e internacional para alcançar resultado robusto.

Quanto ao idioma das produções dos docentes-pesquisadores do campo da Cancerologia brasileira, constatou-se a presença de três idiomas: inglês, português e espanhol. Salta aos olhos o fato de que a maioria das produções, ou seja, 5.916 (97,7\%), foi escrita em inglês. Por outro lado, as produções escritas em português e espanhol somam apenas 141 (2,3\%) do total. Sobre o número significativo de produções em língua inglesa, no campo em análise, pode-se inferir que os pesquisadores do campo possuem, como meta, o estabelecimento do que Mueller (1999) denomina de "núcleo de elite". Este núcleo é formado pelos melhores títulos de periódicos científicos, reconhecidos pelos pesquisadores do campo e que abriga publicações de artigos em língua inglesa, os quais 
alcançam mais visibilidade entre os pesquisadores e, consequentemente, maior número de citações do que os artigos em outras línguas.

Outra categoria de análise das publicações dos docentes-pesquisadores do campo da Cancerologia brasileira foi o seu ano de publicação. Os resultados, expostos no Gráfico 3, apresentam informações interessantes acerca da produtividade do campo, por ano investigado.

Gráfico 3 - Ano de publicação das produções dos docentes-pesquisadores (2005-2015)

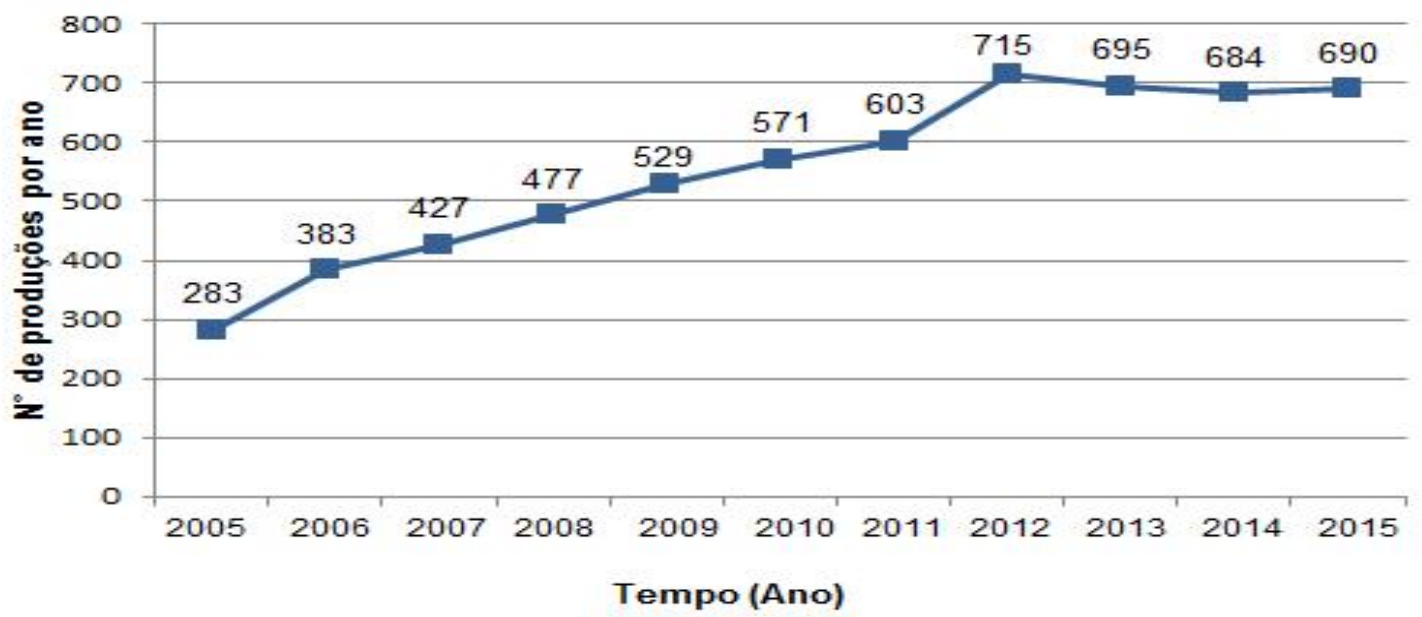

Fonte: Dados da pesquisa.

Observa-se que ocorreu um crescimento de grande importância entre os anos de 2005 a 2012, período semelhante ao do credenciamento de quatro programas de pós-graduação, no campo da Cancerologia brasileira: PPG-INCA (2005), PPG-HCB e PPG-UFPA (2011) e PPG-USP/RP (2012). Estes novos programas podem ter impulsionado um efeito em cadeia: maior número de docentes-pesquisadores, consequentemente, aumento na produtividade do campo científico. Já no período de 2013 a 2015, os resultados revelam redução moderada no número das produções destes docentes-pesquisadores. Estes resultados podem ser interpretados negativamente, uma vez que representam a diminuição na produtividade destes docentes-pesquisadores.

Durante a avaliação da Capes a que são submetidos os programas de pós-graduação brasileira, o item "produção intelectual" é bem pontuado. Nas três últimas avaliações da Capes ocorridas em 2007-2009, 2010-2012 e 2013-2016, o peso conferido a este item sofreu variação entre $30 \%$ e 40\%. (COORDENAÇÃO DE APERFEIÇOAMENTO DE PESSOAL DE NÍVEL SUPERIOR, 2009, 2012, 2016). Ao analisar a relação entre nota de programa e número de publicação, observa-se que o Programa PPG-FAP, com nota cinco, 
superou em publicações o programa PPG-INCA, com nota seis. O resultado desta análise comparativa é possível de ser evidenciado nos Gráficos 4 e 5.

Gráfico 4 - Nota dos programas (2005-2015)

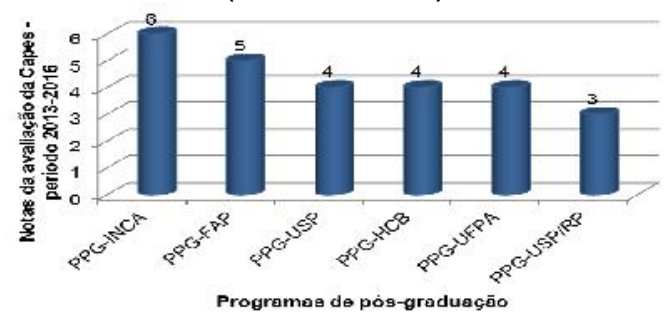

Fonte: Dados da pesquisa.
Gráfico 5 - Produção $0^{(1)}$ dos programas (2013-2016)

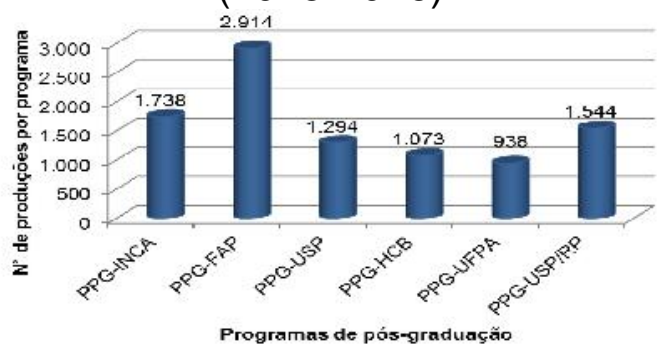

Fonte: Dados da pesquisa.

(1) $O$ total de produções excede 6.057 , porque foram computadas as resultantes de pesquisas em coautoria com docentes-pesquisadores do campo da Cancerologia brasileira.

A média de produção dos programas apresentou relação inversa com o conceito atribuído, na avaliação dos programas,realizada pela Capes, no período de 2013 a 2016. O programa PPG-USP/RP, avaliado com nota 3, apresentou uma média de produção, por ano, correspondente a 140 publicações, enquanto que, nos programas com nota 4 , a média de escritos, por ano, reduziu-se para 100. Semelhante relação foi identificada ao se avaliar as produções dos programas com notas 5 e 6 , pois, enquanto a média anual de publicação do programa com nota 5 foi de 265 produções, por ano, no programa com nota 6, a média anual decai para 158.

Sobre o PPG-FAP e o PPG-INCA, o resultado pode ter relação com o período de credenciamento do programa, assim como com o número de docentes-pesquisadores. O PPG-FAP foi credenciado em 1997 e possui 76 docentes-pesquisadores, enquanto que o PPG-INCA se credenciou em 2005 e possui 51 docentes-pesquisadores. A relação entre tempo de credenciamento e quantidade de produção é pertinente porque, quanto maior o tempo de credenciamento de um programa de pós-graduação, maior a compreensão que os docentes-pesquisadores deste campo alcançarão acerca do funcionamento do campo e do fazer científico deste.

Para analisar a quantidade de publicações entre os docentes-pesquisadores no campo da Cancerologia brasileira, optou-se por estabelecer 11 faixas que variam "de 1a 10" publicações até "Acima de 100" publicações, como pode ser visualizado no Gráfico 6. 
Gráfico 6 - Publicações por docentes-pesquisadores (2005-2015)

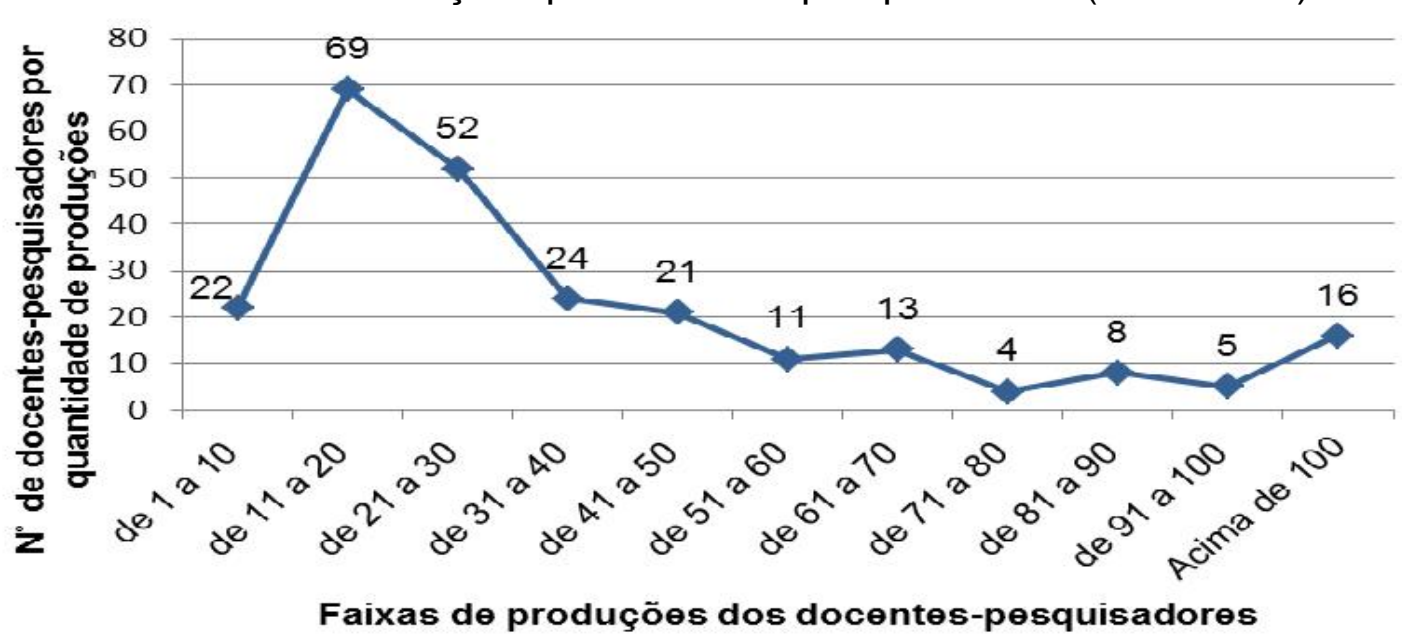

Fonte: Dados da pesquisa.

Observa-se que 69 docentes-pesquisadores (28,2\%) produziram, no período de 2005 a 2015, "de 11 a 20" publicações. Em contrapartida, 4 (1,6\%) docentes-pesquisadores produziram "de 71 a 80 " produtos, no mesmo período. Esses números podem ser lidos à luz da Lei de Lotka ou lei do quadrado inverso, que permite afirmar, para este caso, que poucos pesquisadores produzem muito e a maioria dos pesquisadores produz pouco. Ressalta-se que 16 (6,5\%) docentes-pesquisadores concentram-se na faixa que corresponde à produtividade "Acima de 100".

Esses docentes-pesquisadores demonstram ser, no que concerne ao quesito quantidade, altamente produtivos no campo em análise, e podem ser, também, aqueles que detêm o "monopólio da autoridade científica definida, de maneira inseparável, como capacidade técnica e poder social; ou, se quisermos, o monopólio da competência científica, compreendida enquanto capacidade de falar e agir legitimamente [...]" (BOURDIEU, 1983, p. 122-123, grifo do autor), o que significa dizer, de maneira autorizada e com autoridade entre os seus pares, pois estes docentes-pesquisadores alcançaram o "monopólio da autoridade científica".

A distribuição do Qualis/Capes, dos títulos de periódicos utilizados pelos docentes-pesquisadores para disseminar os resultados de suas pesquisas, no período de 2005-2015, estão dispostos no Gráfico 7 
Gráfico 7 - Distribuição por estrato Qualis/Capes dos títulos de periódicos utilizados como canal de comunicação pelos docentes-pesquisadores (2005-2015)

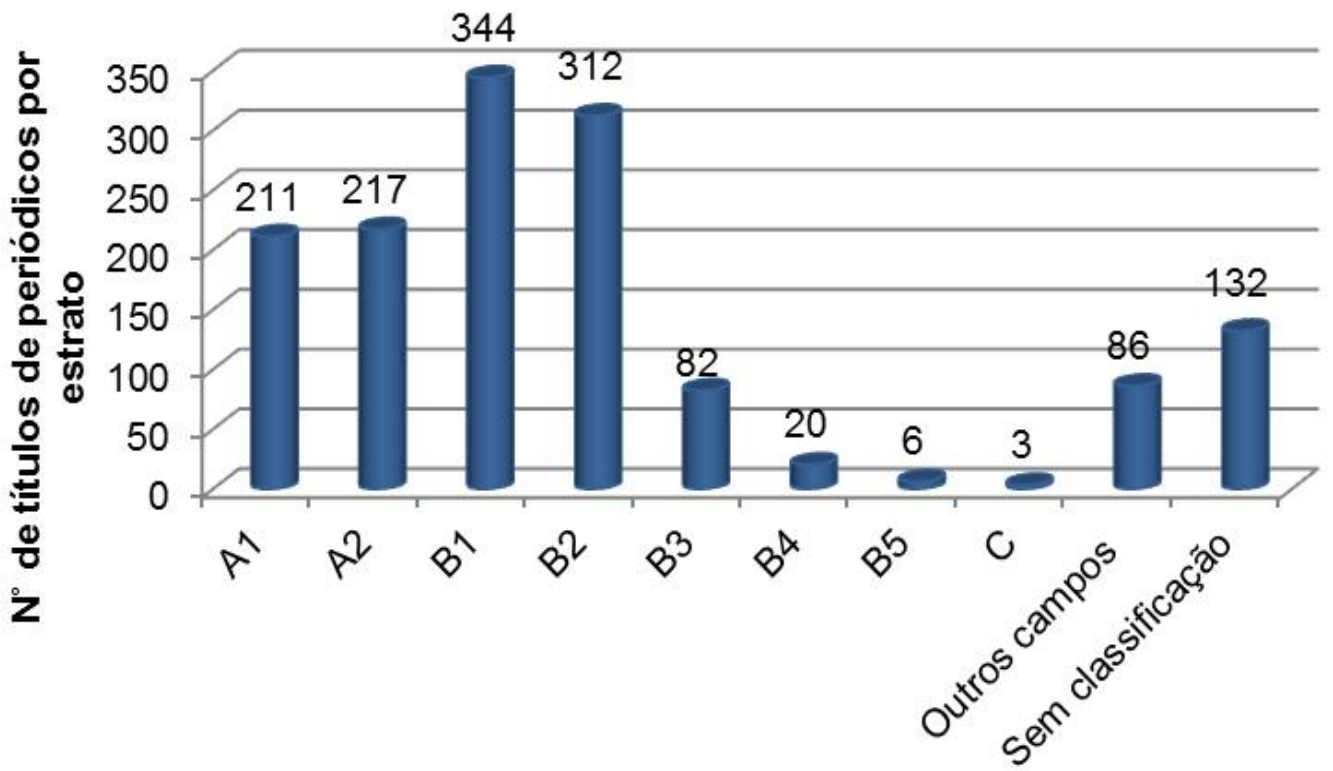

Estratos do Qualis/Capes (2013-2016)

Fonte: Dados da pesquisa.

Em relação aos estratos do Qualis/Capes 2013-2016, os resultados denotam que a maior representatividade dos títulos de periódicos, em que foram publicados os resultados das pesquisas dos docentes-pesquisadores, no campo da Cancerologia brasileira, concentra-se no estrato B1, com 344 (24,3\%) títulos de periódicos científicos. Segundo a comissão de área do sistema de avaliação da Coordenação de Aperfeiçoamento de Pessoal de Nível Superior $(2009,2012,2016)$, este estrato é um dos mais valorizados, juntamente com o A1 e o A2, no campo em análise. Os resultados também evidenciaram que $86(6,1 \%)$ títulos de periódicos não haviam sido classificados em Medicina I, no Qualis/Capes, do período 2013-2016.

Estes títulos de periódicos foram classificados, em outras áreas de avaliação da Capes, a exemplo de: Nutrição; Saúde Coletiva; Psicologia; Enfermagem; Educação Física; Biotecnologia; Medicina II; Odontologia; Interdisciplinar, entre outras áreas de avaliação, e estão reunidos na categoria "Outros campos". A publicação dos resultados das pesquisas dos docentes-pesquisadores, do campo em análise, em títulos de periódicos de áreas, como as elencadas, valida a direção multidisciplinar que os programas de pós-graduação, no campo da Cancerologia brasileira, vêm adquirindo, como recomendação da Coordenação de Aperfeiçoamento de Pessoal de Nível Superior (2016). 
O desafio de publicar os resultados de pesquisa, em títulos de periódicos representativos no campo, é imposto a todos os pesquisadores. Sobre a relevância do título de periódico para o pesquisador, Mueller (1999) esclarece que "o periódico em que o artigo científico é publicado se torna um ponto chave em qualquer carreira científica, pois condiciona as chances da ocorrência de citações a esse artigo.”. Nesse contexto, mais do que tornar públicos os resultados das pesquisas, é importante que eles sejam publicados em um título de periódico reconhecido pelos pesquisadores do campo.

$\mathrm{Na}$ Cancerologia brasileira, os títulos de periódicos classificados no Qualis/Capes, entre os estratos A1, A2 e B1 são os mais importantes. Assim, o ato de tornar públicos os resultados das pesquisas é uma regra no campo da ciência; contudo, onde publicar é uma ação regulada pelo habitus que, ao mesmo tempo em que abre um leque de alternativas de títulos de periódicos científicos que podem ser utilizados para publicar os resultados da pesquisa, aponta para as melhores opções de onde publicar. (BOURDIEU, 2004). Os resultados dessa análise estão distribuídos no Quadro 1, por título de periódico, número de publicações e Qualis/Capes:

Quadro 1 - Títulos de periódicos mais utilizado como canal de comunicação pelos docentes-pesquisadores (2005-2015)

\begin{tabular}{|c|c|c|c|}
\hline Ranking & Títulos de periódicos & $\begin{array}{c}\text { Quant. de } \\
\text { publicações }\end{array}$ & $\begin{array}{l}\text { Qualis/Capes } \\
\text { (2013-2016) }\end{array}$ \\
\hline $1^{\circ}$ & Plos One & 200 & $\mathrm{~B} 1$ \\
\hline $2^{\circ}$ & Brazilian Journal of Medical and Biological Research & 94 & B2 \\
\hline $3^{\circ}$ & Pesquisa Veterinária Brasileira & 72 & Sem classificação \\
\hline $4^{\circ}$ & Clinics & 70 & B2 \\
\hline $5^{\circ}$ & Genetics and Molecular Research & 63 & B3 \\
\hline $6^{\circ}$ & Journal of Clinical Oncology & 54 & A1 \\
\hline $7^{\circ}$ & Bmc Cancer & 53 & $\mathrm{~A} 2$ \\
\hline $8^{\circ}$ & $\begin{array}{l}\text { Head and Neck - Journal for the Sciences and } \\
\text { Specialties of the Head and Neck }\end{array}$ & 53 & Sem classificação \\
\hline $9^{\circ}$ & Arquivos de Neuro-Psiquiatria & 49 & B3 \\
\hline $10^{\circ}$ & Leukemia Research & 45 & B1 \\
\hline $11^{\circ}$ & Genetics and Molecular Biology & 44 & B2 \\
\hline $12^{\circ}$ & Pediatric Blood \& Cancer & 44 & B1 \\
\hline $13^{\circ}$ & International Journal of Cancer & 37 & $\mathrm{~A} 1$ \\
\hline $14^{\circ}$ & Blood & 34 & $\mathrm{~A} 1$ \\
\hline $15^{\circ}$ & Cancer Genetics and Cytogenetics & 33 & B4 \\
\hline $16^{\circ}$ & Oral Oncology & 33 & A2 \\
\hline
\end{tabular}

Fonte: Dados da pesquisa.

Ao analisar os 16 títulos de periódicos mais utilizados pelos docentes-pesquisadores do campo, para publicar os resultados de suas pesquisas, o ranking revela que a quantidade de títulos de periódicos classificados no Qualis/Capes 2013-2016, 


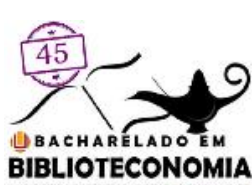

entre os estratos $A 1, A 2$ e $B 1$, representa a mesma proporção dos títulos de periódicos nos estratos B2; B3; B4; B5; C e "Sem classificação", 8 (50,0\%) títulos de periódicos para cada grupo.

Quanto à quantidade de publicações disseminadas nos títulos de periódicos, observa-se que a dimensão é quase a mesma entre ambos os grupos de estratos Qualis/Capes. Isso, pois, 500 (51,1\%) títulos de periódicos foram classificados nos estratos A1, A2 e B1 e 478 (48,9\%), no segundo grupo de classificação.

\section{CONSIDERAÇÕES FINAIS}

Os resultados apresentam indícios de que as contribuições científicas, acumuladas dos docentes-pesquisadores ao longo de sua carreira acadêmica, vêm contribuindo para a consolidação, no que se refere à produção científica do campo da Cancerologia brasileira. Observou-se, ainda, que houve um crescimento exponencial entre 2005 e 2012, alcançando, no ano de 2012, 715 (11,8\%) publicações. Nos anos subsequentes, apesar de leve declínio no número de publicações científicas, elas mantiveram-se acima de 680.

Quanto às características da produção científica dos docentes-pesquisadores do campo da Cancerologia brasileira, observou-se a predominância de artigos científicos publicados em periódicos científicos. Esta característica pode estar atrelada ao fato de que, na orientação da comissão de avaliação da Capes, indica-se o artigo publicado em periódico científico, como um dos principais indicadores da produção intelectual no campo. (COORDENAÇÃO DE APERFEIÇOAMENTO DE PESSOAL DE NÍVEL SUPERIOR, 2009, 2012, 2016).

Outro fator a ser destacado sobre as investigações no campo é o uso do inglês enquanto idioma das publicações, pois quase a totalidade das produções foi publicada neste idioma. Sobre os estratos dos periódicos científicos, evidenciou-se que 3.604 produções $(60,0 \%)$ dos docentes-pesquisadores do campo concentraram-se em periódicos classificados no Qualis/Capes 2013-2016, nos estratos A1, A2 e B1. Isso reafirmou a qualidade dos periódicos científicos, visto que tais estratos são os mais representativos no processo de avaliação da Capes.

É oportuno sinalizar que os objetivos da pesquisa foram alcançados. Entretanto, sugere-se a realização de novos esforços investigativos, a fim de identificar possíveis 
alterações nos padrões de comunicação dos resultados da pesquisa. Recomenda-se, também, a realização de pesquisas em outros campos científicos, para que seja possível uma melhor compreensão do processo de comunicação entre os docentes-pesquisadores.

\section{REFERÊNCIAS}

ANDRIOLO, A. et al. Classification of journals in the Qualis system of CAPES - urgent need of changing the criteria! Jornal Brasileiro de Pneumologia, São Paulo, v. 36, n. 1, p. 1-3, jan./fev. 2010. Disponível em: http://www.scielo.br/pdf/jbpneu/v36n1/en_v36n1a01.pdf. Acesso em: 14 mar. 2013. ASSOCIAÇÃO BRASILEIRA DE NORMAS TÉCNICAS. NBR 6022: informação e documentação: artigo em publicação periódica científica impressa: apresentação. Rio de Janeiro, 2003.

BARATA, R. de C. B. Dez coisas que você deveria saber sobre o Qualis. Revista Brasileira de Pós-Graduação, Brasília, DF, v. 13, n. 30, p. 013-040, jan./abr. 2016. Disponível em: http://ojs.rbpg.capes.gov.br/index.php/rbpg/article/view/947/pdf. Acesso em: 30 mar. 2016 BOURDIEU, P. O campo científico. In: ORTIZ, R. (org.). Pierre Bourdier: sociologia. São Paulo: Ática, 1983. p. 122-155.

BOURDIEU, P. Os usos sociais das ciências: por uma sociologia clínica do campo científico. Tradução de Denice Barbara Catani. São Paulo: UNESP, 2004. 87p.

BOURDIEU, P. Para uma sociologia da ciência. Lisboa: Edições 70, 2014. (Coleção Biblioteca, 70). BUFREM, L. S. Relações interinstitucionais e autoria em artigos de revistas científicas de Ciência da Informação no Brasil. In: ENCONTRO NACIONAL DE PESQUISA EM CIÊNCIA DA INFORMAÇÃO, 10., 2009, João Pessoa, PB. Anais [...]. João Pessoa: UFPB, 2009. Disponível em: http://enancib.ibict.br/index.php/enancib/xenancib/paper/viewFile/3299/2425. Acesso em: 25 out. 2016.

CAMPOS, J. N. B. Qualis periódicos: conceitos e práticas nas Engenharias I. Revista Brasileira de Pós-Graduação, Brasília, DF, v. 7, n. 14, p. 477-503, dez. 2010. Disponível em: file://C:/Users/katia_000/Downloads/14-32-1-SM.pdf. Acesso em: 15 fev. 2012.

COORDENAÇÃO DE APERFEIÇOAMENTO DE PESSOAL DE NÍVEL SUPERIOR. Documento de área Medicina I. 2009. Disponível em:

http://www.capes.gov.br/images/stories/download/avaliacao/MED_I20nov09q.pdf. Acesso em: 05 ago. 2012.

COORDENAÇÃO DE APERFEIÇOAMENTO DE PESSOAL DE NÍVEL SUPERIOR. Documento de área Medicina I. 2012. Disponível em:

http://www.capes.gov.br/images/stories/download/avaliacaotrienal/Docs_de_area/Medicina_I_doc_ area_e_comiss\%C3\%A3o_att08deoutubro.pdf. Acesso em: 05 ago. 2014.

COORDENAÇÃO DE APERFEIÇOAMENTO DE PESSOAL DE NÍVEL SUPERIOR. Documento de área Medicina I. 2016. Disponível em:

http://capes.gov.br/images/documentos/Documentos_de_area_2017/15_MED_I_docarea_2016.pdf. Acesso em: 05 ago. 2016.

COORDENAÇÃO DE APERFEIÇOAMENTO DE PESSOAL DE NÍVEL SUPERIOR. Diretória de Avaliação. Qualis: concepção e diretrizes básicas. Revista Brasileira de Pós-Graduação, Brasília, DF, v.1, n. 1, p. 149-151, jul. 2004.

GARVEY, W. D. Communication: the essence of Science. Oxford: Pergamon, 1979.

LETA, J. Análise da produção científica a partir de indicadores bibliométricos. In: LANDI, Francisco Romeu; GUSMÃO, Regina (coord.). Indicadores de ciência, tecnologia e inovação em São Paulo2004. São Paulo: FAPESP, 2005. p. Disponível em:

http://www.fapesp.br/indicadores2004/volume1/cap05_vol1.pdf. Acesso em: 15 out. 2015. 


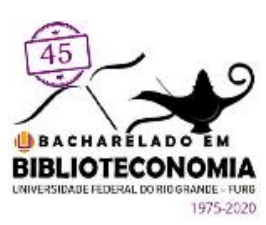

MEADOWS, A. J. A comunicação científica. Tradução de Antonio Agenor Briquet de Lemos. Brasília, DF: Briquet de Lemos, 1999.

MUELLER, S. P. M. A ciência, o sistema de comunicação científica e a literatura científica. In: CAMPELLO, B. S.; CENDÓN, B. V.; KREMER, J. (org.). Fontes de informação para pesquisadores e profissionais.Belo Horizonte: Ed. UFMG, 2007a. p. 21-34.

MUELLER, S. P. M. A publicação da ciência: áreas científicas e seus canais preferenciais. Data Grama Zero - Revista de Ciência da Informação, Rio de Janeiro, v. 6, n. 1, fev. 2005. Disponível em: http://repositorio.unb.br/bitstream/10482/980/2/ARTIGO_PublicacaoCiencia.pdf. Acesso em: 13 jun. 2015.

MUELLER, S. P. M. Literatura científica, comunicação científica. In: TOUTAIN, L. M. B. B. (org.). Para entender a ciência da informação. Salvador: Edufba, 2007b. (Sala de aula; 6).

MUELLER, S. P. M. Métricas para a ciência e tecnologia e o financiamento da pesquisa: algumas reflexões. Encontros Bibli: Revista Eletrônica de Biblioteconomia e Ciência da Informação, Florianópolis, n. esp. 1. sem. p. 24-35. 2008. Disponível em:

https://periodicos.ufsc.br/index.php/eb/article/view/1518-2924.2008v13nesp1p24/1593. Acesso em: 16 set. 2015.

MUGNAINI, R. Avaliação da produção científica nacional: contextualização e indicadores. In: POBLACIÓN, D. A. et al. Revista científica: dos processos às perspectivas alternativas de comunicação. Cotia: Ateliê, 2011. P. 43-68.

MUGNAINI, R.; MENEGHINI, R.; PACKER, A. Citation profiles in Brasilian journals of the SciELO databasse in different scientific areas. In: INTERNATIONAL CONFERENCE OF THE INTERNATIONAL SOCIETY FOR SCIENTOMETRICS AND INFORMTRICS, 11., 2007, Madrid. Proceedings [...] Madrid, 2007. Disponível em:

https://www.researchgate.net/publication/275274284_Citation_profiles_in_Brazilian_journals_of_the_ SciELO_database_in_different_scientific_areas. Acesso em: $15 \mathrm{dez} .201 \overline{5}$.

NEDERHOF, A. J. Bibliometric monitoring of research performance in the Social Sciences and the Humanities: a review. Scientometrics, Amsterdam, v. 66, n. 1, p. 81-100, 2006. Disponível em: https://link.springer.com/content/pdf/10.1007\%2Fs11192-006-0007-2.pdf. Acesso em: 10 jan. 2016. ZIMAN, J. Conhecimento público. Tradução de Regina Regis Junqueira. São Paulo: Itatiaia: USP, 1979. 